\title{
Visualization of Spectral Images
}

\author{
Gerrit Polder $^{a, b}$ and Gerie W.A.M. van der Heijden ${ }^{a}$ \\ ${ }^{a}$ Plant Research International, PO-Box 16, 6700 AA, Wageningen, The Netherlands \\ ${ }^{b}$ Delft University of Technology, Faculty of Applied Physics, Pattern Recognition Group, \\ Lorenzweg 1, 2628 CJ, Delft, The Netherlands
}

\begin{abstract}
Spectral image sensors provide images with a large number of contiguous spectral channels per pixel. Visualization of these huge data sets is not a straightforward issue. There are three principal ways in which spectral data can be presented; as spectra, as image and in feature space. This paper describes several visualization methods and their suitability in the different steps in the research cycle. Combinations of the three presentation methods and dynamic interaction between them, adds significant to the usability. Examples of some software implementations are given. Also the application of volume visualization methods to display spectral images is shown to be valuable.
\end{abstract}

Keywords: Spectral images, feature space, volume visualization

\section{INTRODUCTION}

Recent developments in optics and sensors allow imaging spectrometry: the creation of spectral images, i.e. images with wavelength-specific measurements over a large part of the spectrum. ${ }^{1,2}$ In relation to gray-value images, these images are an order of magnitude larger. Besides a 2D spatial dimension of e.g. 256x256 pixels, a spectral image also has a third spectral dimension of e.g. 256 wavelengths. In literature the terms multispectral and hyperspectral imaging are often used. Multispectral is mostly used for images with 10-100 wavelength bands, where hyperspectral is used for images with more than 100 bands. In this paper we do not make this distinction, we use the term spectral image independent of the number of wavelength bands.

Spectral imaging is commonly used in the area of remote sensing, where images of the earth surface are taken from satellite, or from an airplane. ${ }^{3,4}$ Recently cheap and easy to use sensors came available, and now this technique is more and more used on macroscopic and microscopic scale. This paper focuses on the latter two. Spectral images can be described as a three-dimensional data cube. Visualization of these data is not a straightforward issue. More complex data require more complex and sophisticated data analysis procedures if their full potential is to be achieved. The matter of how the variations are represented mathematically and conceptually is an important first step in defining how the analysis process should proceed. In fact each step in the research cycle, comprising data gathering, data exploration, data analysis and presentation of results, has specific requirements for the visualization of spectral image data. In table 1 the research cycle steps related to their visualization requirements are tabulated.

Landgrebe $^{5}$ distinguished three principal ways in which spectral images can be represented quantitatively:

- As spectra, which shows variations of a pixel or region as a function of wavelength

- In image form, where pixels are displayed in geometric relationship to one another

- In feature space, where pixels are displayed as points in an N-dimensional space

In this paper we will present several visualization methods for these data representations and give examples of their use, related to data analysis. Also visualization methods as a combination of different data representation modes are discussed, including volume rendering and ray tracing techniques. The paper is organized as follows. First spectral, image and feature based representation of spectral images are described. Then some examples of visualization methods and software are given. We end the paper with discussion and some concluding remarks.

Further author information: (Send correspondence to G. Polder)

G. Polder: E-mail: G.Polder@plant.wag-ur.nl

G.W.A.M. van der Heijden: E-mail: G.W.A.M.vanderHeijden@plant.wag-ur.nl 
Table 1. Visualization requirements of spectral image data related to the steps in the research cycle.

\begin{tabular}{|l|l|l|l|}
\hline & Step & Purpose & Visualization requirements \\
\hline \hline 1 & Data gathering & First data screening & Speed and easy of use \\
\hline 2 & Data exploration & Generation of hypotheses & Versatile and flexible, interaction needed \\
\hline 3 & Data analysis & Pattern recognition techniques & Versatile and flexible, no interaction \\
\hline 4 & Presentation & Presentation and publication of the results & Eye-catching, informative picture \\
\hline
\end{tabular}

\section{SPECTRAL BASED REPRESENTATION}

In the research cycle spectral based representation is most often used at the data gathering and the data exploration step. From this finding it is obvious that many algorithms for the analysis of spectral images, which appear in literature, begin with a representation of a response function as a function of wavelength. Identifiable features within a spectral response function, such as absorption bands due to resonances at the molecular level, can be used to identify compounds associated with a given spectrum. This approach, arising from the concepts of chemical spectroscopy, which has long been used in the laboratory for molecular identification, is perhaps one of the most fundamentally cause/effect based approaches to the analysis of spectral images. Traditionally spectroscopy analysis techniques are used to analyze each pixel in the image, without using the spatial relationship. From groups of pixels, which are related in spatial or spectral sense, the mean spectra with its confidence limits can be calculated. The spectra are visualized using a two-dimensional graph of the wavelength verses the absorption or reflection value, sometimes extended with an indication of dispersion using error bars. Pure spectral representation is not used in the last step in the research cycle, but combinations of spectral representation with image or feature based representation can be useful for presentation.

\section{IMAGE BASED REPRESENTATION}

Image based representation is important in all steps in the research cycle. Therefore the image form is perhaps the first form one thinks of when considering spectral imaging as a source of information. Indeed, the spatial relationship between the spectra is crucial for most applications. However, for classification purposes, it is not so straightforward to maintain spatial relationship when performing spectral analysis, e.g. before decomposing spectra in principal components, the image is unfolded resulting in a one-dimensional array of spectra, disregarding the spatial relationship between the data. ${ }^{6}$ Nevertheless data in image form serve as the human/data interface in that image space helps the user to make the connection between individual pixel areas and the surface features they represent. To display a spectral image, a first step would be to reduce it into a two-dimensional gray value image, e.g. by taking the mean of the wavelength values, or by taking a specific wavelength band from the data. The data can also be reduced to a RGB color image (which in the strict sense is a spectral image with 3 bands) by taking three specific bands from the spectral image, or by applying a weigh function to the wavelength data. In these cases a false color image is produced. When the wavelengths of the spectral image cover the visible range, sensible weights are the spectral tristimulus values as defined for a standard colorimetric observer by CIE (1964). In this case a true color image is produced.

\section{FEATURE BASED REPRESENTATION}

Often spectral image analysis is used to automatically find and measure objects in the image. Pattern recognition techniques are used to extract the features from the data. In the research cycle this is done in the data exploration and the data analysis steps. For the presentation step, feature based presentation is crucial. Feature based representation begins with a spectral focus, but it is less related to pictures or graphs. The sampled discrete spectrum of a pixel can be regarded as a feature vector in an $\mathrm{N}$-dimensional space, where $\mathrm{N}$ is the number of spectral bands. This conversion of the information from a continuous function of wavelength to a discrete point in a vector space is not only inherent in the operation of a spectral sensor, it is a crucial representation for pattern recognition, i.e. analysis by a machineimplemented algorithm. High dimensional spaces can not be visualized directly. Visualization is restricted to only a few (typically 2 or 3 ) dimensions. Therefore, from a visualization point of view, the N-dimensional vector needs to be reduced by the selection or combination of features in order to visualize it in two or three dimensional scatterplots. 


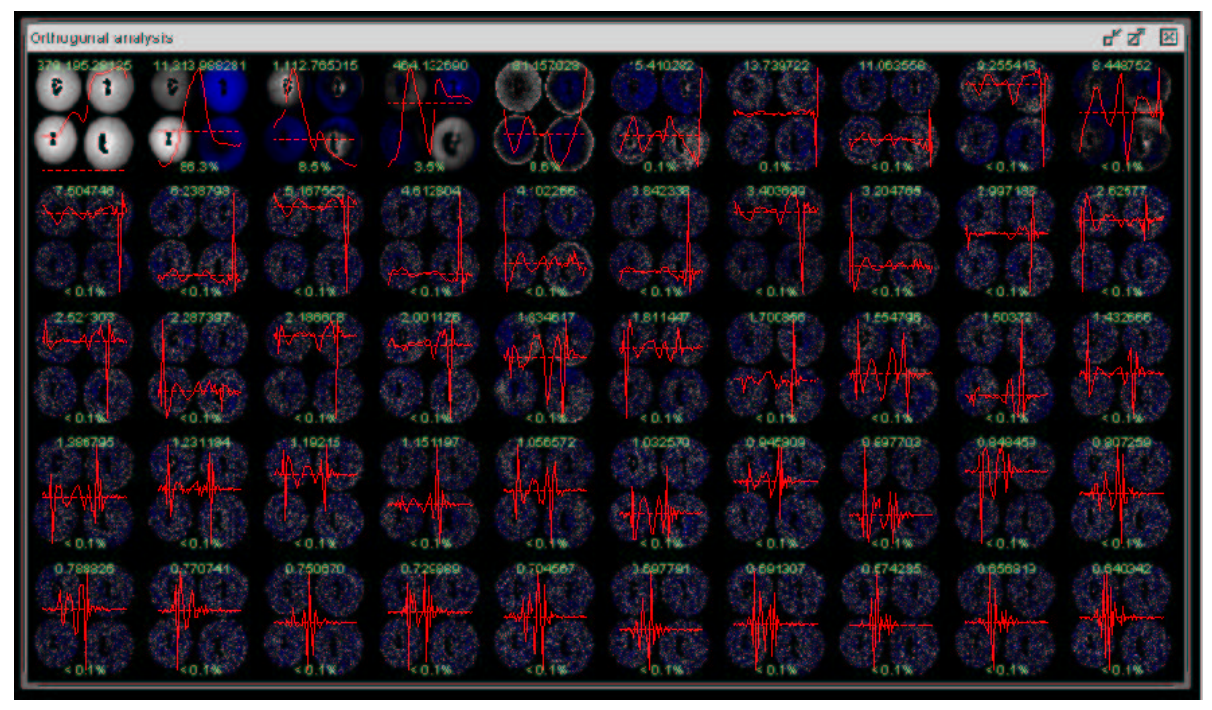

Figure 1. Trellis plot of PCA analysis of four tomatoes which differ in maturity. The eigenimages, eigenvectors and eigenvalues are shown.

Several strategies and criterion functions are possible for searching good subsets. One of the most well known and often used methods is Principal Component Analysis. In PCA the original feature vector is decomposed into a new set of features, being linear combinations of the original features, with the addition that the first few features contain most of the variation present in the original set. Feature reduction can be obtained by selecting only the first $M$ features where $M \ll N$. The most important scores or loadings can be visualized in a two- or three-dimensional graph. ${ }^{6}$ In addition to the visualization possibilities and an improved computational speed in lower dimensional feature spaces there might also be an increase in the accuracy of the classification algorithms. ${ }^{7,8}$ This is caused by the fact that for smaller feature sets less parameters have to be estimated. Besides PCA, other ways exist to reduce the dimensionality by mapping the data on a linear or nonlinear subspace. Examples of these techniques are Fisher linear or quadratic discriminant analysis, independent component analysis, neural networks and support vector machines.

\section{COMBINING SPECTRAL, IMAGE AND FEATURE BASED REPRESENTATION}

Combinations of the three presentation methods and dynamic interaction between them, adds significant to the usability. This section describes some examples.

\subsection{Combination of image and spectral display}

In the data gathering and data exploration steps, both spectral and image based representation have a valuable contribution. The combination of these two representation modes is even more valuable, when data visualized in one form can be linked interactively with data shown in the other form. Isaac (Imaging Spectroscopy Acquisition and Control) (see http://www.ph.tn.tudelft.nl/ polder/isaac.html) is a system for recording and screening spectral images. It consists of specialized hardware and software for the acquisition of spectral images on a macroscopic scale. The main purpose of the software is not visualization of the acquired images, but some simple tools are provided, in order to quickly evaluate the scanned images. The spatial information of different spectral bands can be displayed concurrently. Furthermore of each pixel, the spectral information can be shown. There is a close interaction between the two visualization methods: while clicking or dragging in the image window, the spectrum of the selected pixel is shown. The wavelength plane shown can be selected with a slider. Also tools for contrast stretching the image are provided.

\subsection{Combination of image and feature display}

Since the spatial relationship of the measured features is important, the combination of image and feature display is important in the data exploration, data analysis and the presentation step. By considering a spectral image as an 

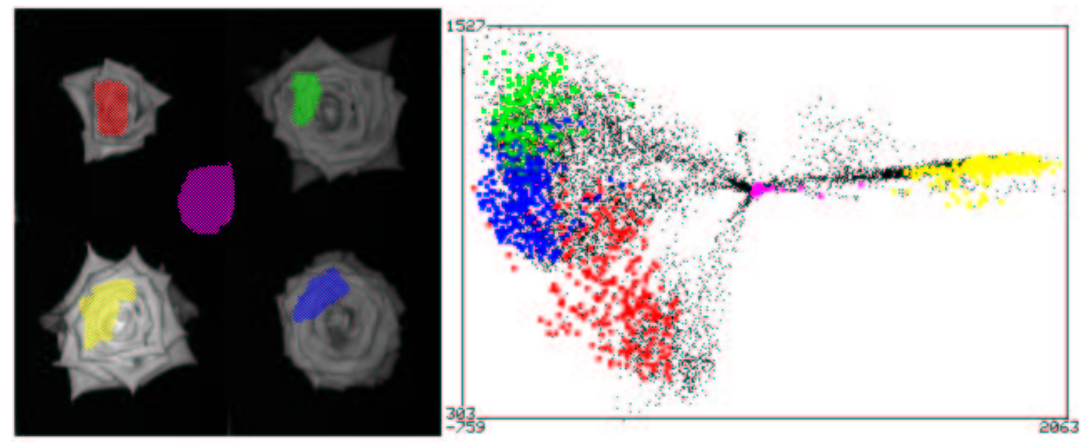

Figure 2. Spectral image of four roses. On the left the first PC component, on the right the PCA score plot of scores 1 and 2. Interactive selections are made in the image, which results in the corresponding scores in the score plot.
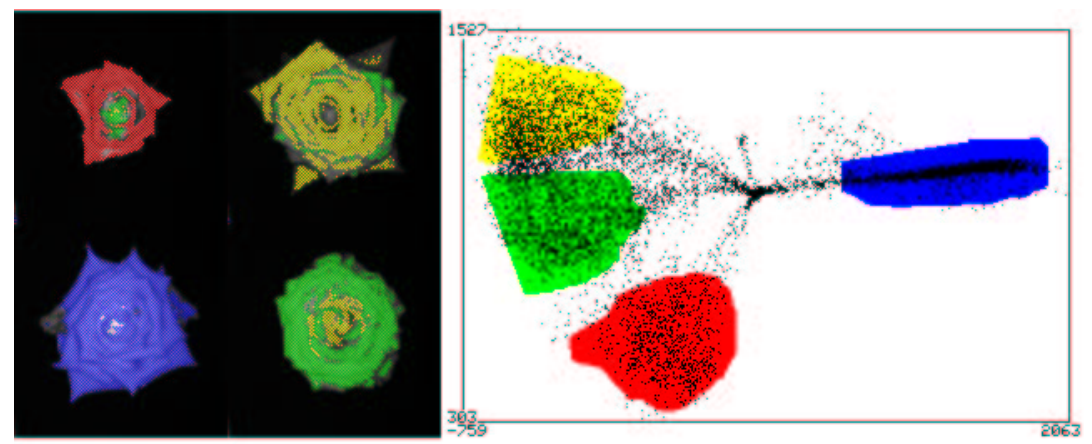

Figure 3. The same spectral image as in figure 2. Interactive selections are made of clusters in the score plot, pixels within this cluster are marked in the image window.

image sequence, a powerful analysis and visualization tool is obtained. Visualization of spatial bands is obtained by showing the image in a Trellis plot, i.e. showing the image at different spectral bands in a grid. Since there are a large number of bands, it is not possible to display each plane in its original size. Figure 1 shows a trellis plot of PCA analysis on a spectral image of tomatoes of different maturity. Besides the score-images, also the eigenvalues and eigenvectors are shown for each principal component. This figure is made with Pixies (Physiological Information eXtraction from ImagE Sequences), by Apteryx, which is a user-friendly application dedicated to the analysis of biomedical image series. A detailed description can be found at http://www.apteryx.fr/en/pixies/. These examples display the data in a static way.

Dynamic interaction between the two visualization methods adds significant to the value. In figure 2 and 3 a spectral image of four different roses is shown. On the left is the sum image of all the wavelength bands, on the right a score plot from score 1 and 2 of the PCA analysis is plotted. In figure 2 regions in the image are selected interactively. The corresponding scores are marked in the score plot. Figure 3 shows the other way around, the corresponding pixels of selected clusters in the score plot are marked in the image. Additionally zooming and panning tools are provided. This interactive visualization method was accomplished with in-house software written in $\mathrm{C}$ and implemented in Scil-Image (TNO-TPD, Delft, NL).

\subsection{Volume visualization techniques applied to spectral images}

Since a spectral image is organized as a 3D sampled scalar field, standard volume visualization techniques can be applied to visualize the data. In contrast with real 3D images (e.g obtained with Magnetic Resonance Imaging of Confocal Scanning Laser Microscopy) a voxel does not represent a 3D spatial point, instead it represents the reflection value of a 2D spatial point at a certain wavelength. Figure 4 shows a combination of slices of a spectral image of two flowers of Saint Paulia, violet and pink. The violet flower has much lower reflection values in the lower wavelength 

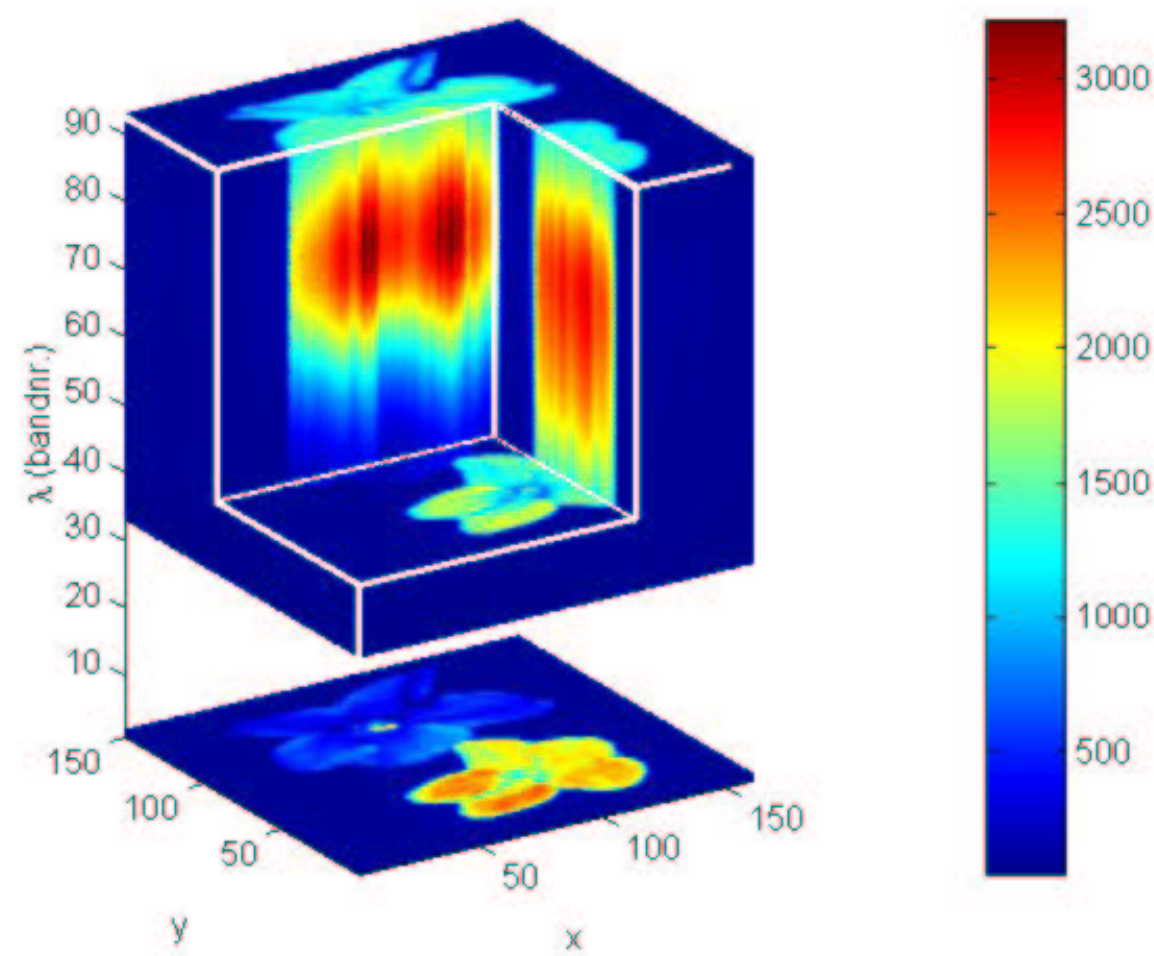

Figure 4. Direct volume rendering of a spectral image of a violet (upper left) and a pink (lower right) flower of Saint Paulia. To visualize several aspects of this image, a cross-section at 75 in the spectral direction is cut out. At the bottom plane number 50 is displayed.

bands. Spatial information is preserved, but only at the selected cross-section. Since a voxel in a spectral image does not represent a spatial 3D data point it was not to be expected that iso-surfaces would be a useful representation method. But due to the fact that in most images there is some shading from the border to the center of the objects this technique gave unexpectedly good results (figure 5). The volume visualization in this section is all done in MATLAB (The Mathworks inc.).

\section{DISCUSSION}

A number of methods can be used to visualize spectral images. All these methods make use of one or more combinations of the spectral, spatial and feature nature of the data. It is difficult to visualize all the aspects of a spectral image in one picture, because of the large size of the data. Displaying one or more image bands gives information on the spatial information in the selected band, but the relation between spectral and spatial information is more difficult to investigate. Adding interactive control, such as selecting a certain band with a slider, or showing the spectrum at selected pixels contributes to the explorative value of this visualization method. Displaying all image bands at once gives more information about the spectral relationship of the image bands. Since the images need to be reduced significantly to fit on the screen, it is difficult to see details in the spatial information. Mapping the features to lower dimensional space (figure 1) can also contribute to the understanding of the data. From spectral band 5 onwards the data contains mainly noise, so displaying image 1-4 at their original sizes gives most of the information. Adding user-interaction contributes significant, as can be seen from figure 2 and 3 . Display of pixels in either image or feature space as a result of interactive selection of points in the other space is useful while exploring the data. Volume visualization techniques can be applied to visualize combinations of spectral and spatial information (figure 4). Both spectral and spatial characteristics are shown in detail, but only at the selected cross-section. To explore the whole data set, interactive selection of cross-sections is needed and real-time update of the display is 


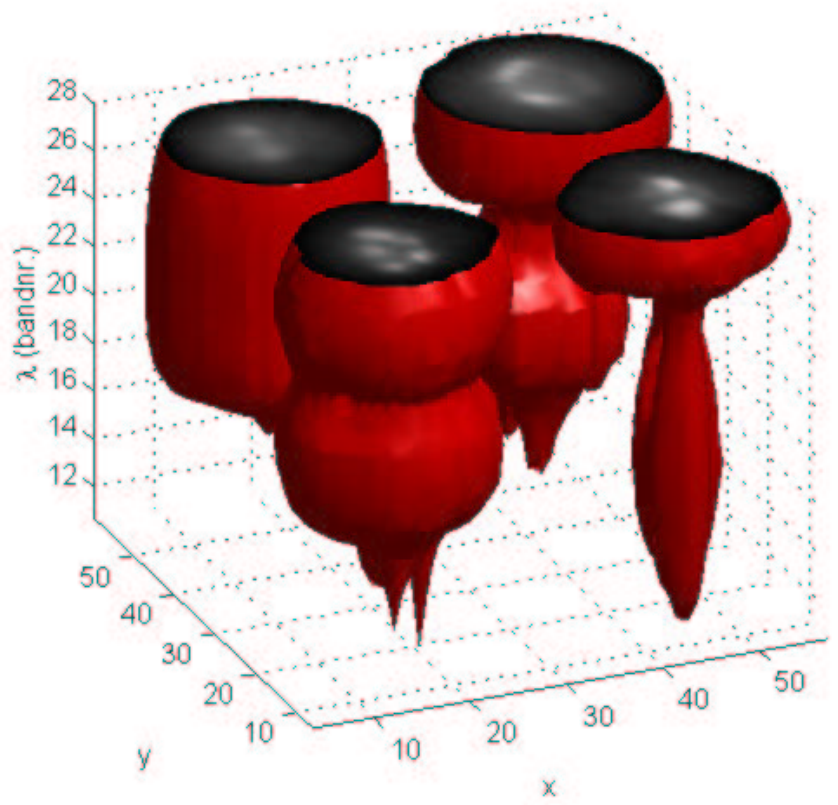

Figure 5. Iso-surface of a spectral image of four tomatoes of different maturity. The lower right one is the greenest one, then upper right, lower left and the upper left the ripest. Using a cross section the reflection values at wavelength band 28 are also visualized.

desirable. This also applies to rotating the data set, the viewpoint or the illumination direction. The same accounts for iso-surfaces where the threshold level (iso-contour) needs to be selected in an interactive way, with fast update of the display.

For presentation of data, an important aspect is to gather as much information in a single picture as possible without losing insight. Figure 4 shows a nice example of combining the spectral information at interesting crosssections, with a spatial representation in the form of an isocap. Although at the moment hardly possible, user interaction could also be useful for presentation purposes. For presentation on the internet compression algorithms for spectral images and VRML-browsers with volume rendering capability are required.

\section{REFERENCES}

1. E. Herrala and J. Okkonen, "Imaging spectrograph and camera solutions for industrial applications," International Journal of Pattern Recognition and Artificial Intelligence 10(1), pp. 43-54, 1996.

2. T. Hyvärinen, E. Herrala, and A. Dall'Ava, "Direct sight imaging spectrograph: a unique add-on component brings spectral imaging to industrial applications," in SPIE symposium on Electronic Imaging, vol. 3302, 1998.

3. A. F. H. Goetz, G. Vane, J. E. Solomon, and B. N. Rock, "Imaging spectrometry for earth remote sensing," Sience 228, pp. 1147-1153, 1985.

4. G. Vane and A. F. G. Goetz, "Terrestrial imaging spectrometry: Current status, future trends," remote Sensing of Environment 44, pp. 117-126, 1993.

5. D. Landgrebe, "On information extraction principles for hyperspectral data, a white paper," tech. rep., School of Electrical\& Computer Engineering, Purdue University, 1997.

6. P. Geladi and H. Grahn, Multivariate image analysis, John Wiley \& Sons, New York, 1996.

7. B. D. Ripley, Pattern Recognition and Neural Networks, Cambridge University Press, Cambridge, 1996.

8. R. P. W. Duin, PRTools, Version 3.0, A Matlab Toolbox for Pattern Recognition. Delft, 2000. 